\title{
Polarization dependence of the fundamental absorption edge and the Urbach rule in $\mathrm{PbMoO}_{4}$ crystals
}

\author{
Masami Fujita ${ }^{*, 1}$ and Minoru Itoh ${ }^{* *, 2}$ \\ ${ }^{1}$ Japan Coast Guard Academy, Wakaba, Kure 737-8512, Japan \\ 2 Department of Electrical and Electronic Engineering, Shinshu University, Nagano 380-8553, Japan
}

Received ZZZ, revised ZZZ, accepted ZZZ

Published online ZZZ (Dates will be provided by the publisher.)

PACS 71.35.Cc, 78.20.Ci, 71.35.Aa, 78.40.-q

* Corresponding author: e-mail fujita@jcga.ac.jp, Phone: +81 82321 4961, Fax: +81 823218105

** e-mail itohlab@shinshu-u.ac.jp, Phone: +81 26269 5261, Fax: +81 262695220

The fundamental absorption tail of $\mathrm{PbMoO}_{4}$ crystals has been investigated in the $a c$ plane in a wide temperature range between 6 and $300 \mathrm{~K}$ by using linearly polarized light. A remarkable dichroism between $\boldsymbol{E} / / \boldsymbol{a}$ and $\boldsymbol{E} / / \boldsymbol{c}$ is observed in the tail region. A defect-related absorption band is clarified for the polarization $\boldsymbol{E} / / \boldsymbol{a}$. It is found that the Urbach rule holds for the absorption spectra measured with light polarized with $\boldsymbol{E} / / \boldsymbol{c}$. The results of the analysis of the Urbach tail are discussed in comparison with the experimental results on the exciton transition and luminescence properties in this material.
1 Introduction Lead molybdate $\left(\mathrm{PbMoO}_{4}\right)$ is well known as a superior medium for acousto-optic devices [1, 2]. This material also attracts attention because of its great potential to be used as an effective cryogenic detector for double $\beta$ decay experiments [3]. Therefore the optical absorption [4-10] and the luminescence properties $[4,6,11$, 12] have been studied by many researchers.

$\mathrm{PbMoO}_{4}$ crystallizes in the scheelite structure [13, 14]. The crystal is optically uniaxial with the optical axis along the $c$-axis, and the $a$ - and $b$-axes are equivalent to each other. Recently we have investigated the electronic structure of $\mathrm{PbMoO}_{4}$ by the measurements of polarized reflectivity spectra and X-ray photoemission spectrum, together with the theoretical calculation [15]. It was found that the lowest exciton band exhibits distinct dichroism, from which we proposed that the exciton transition can be explained in terms of the intra-cationic excitation in $\mathrm{Pb}^{2+}$ ions under the uniaxial crystal field along the $c$-axis.

For various non-metallic solids, it has been observed that the fundamental absorption tail obeys the Urbach rule, where the absorption coefficient increases exponentially with increasing photon energy, i.e. logarithms of the absorption coefficient plotted as a function of the photon energy can be approximated by a straight line, and the straight lines for various temperatures cross at one and the same point [16]. The analysis of the Urbach tail gives us important information on the exciton-phonon interaction in the material. van Loo has measured the fundamental absorption tail of $\mathrm{PbMoO}_{4}$ at various temperatures and concluded that the Urbach rule holds for this material [4]. In his study, however, the polarization dependence of the absorption intensity was not taken into account, although the optical constants primarily depend on the polarization in anisotropic crystals such as $\mathrm{PbMoO}_{4}$. Therefore, careful examinations of the absorption-edge spectra using polarized light are necessary to obtain more reliable information on absorption tail of $\mathrm{PbMoO}_{4}$. In the previous work [17], we have measured polarized absorption spectra of $\mathrm{PbWO}_{4}$, which also crystallizes in the scheelite structure, and found the validity of the Urbach rule for this material.

In the present study, we have measured the fundamental absorption spectra of $\mathrm{PbMoO}_{4}$ crystals with use of polarized light in order to clarify the optical anisotropy in the absorption-edge region and to examine whether the Urbach rule holds for this material or not.

2 Experiment $\mathrm{PbMoO}_{4}$ samples used in the present study were purchased from Furukawa Company in Japan. 
The crystal was grown by the Czochralski technique from the raw materials of $\mathrm{PbO}$ of $99.99 \%$ purity and $\mathrm{MoO}_{3}$ of $99.9 \%$ purity in 1:1 molar ratio, and was purified by threetime crystallization. The crystal orientation was determined by the x-ray diffraction. The sample surfaces containing the $a c$ plane were mechanically polished. The thickness of the samples was $2.0 \mathrm{~mm}$. The optical experiments were carried out using linearly polarized light of the synchrotron radiation through a $1 \mathrm{~m}$ Seya-Namioka type monochromator at the beamline $1 \mathrm{~B}$ in UVSOR facility of the Institute for Molecular Science in Okazaki. The samples were mounted on the copper holder in a variable temperature cryostat of He-flow type. Optical transmittance spectra were measured at various temperatures between 6 and 300 $\mathrm{K}$. The temperature of the samples was slowly changed at a rate less than $2.5 \mathrm{~K} / \mathrm{min}$ to avoid the cracking due to rapid temperature change [9]. In order to estimate the reflection losses in the transmittance spectrum at each temperature, the linear portion of the spectrum in the low-energy region with negligible absorption was extrapolated by a straight line through the absorbing region. The absorption-edge spectra were obtained by correcting the reflection losses thus estimated.

3 Results The absorption-edge spectra for the polarization parallel to the $a$-axis $(\boldsymbol{E} / / \boldsymbol{a})$ and $c$-axis $(\boldsymbol{E} / / \boldsymbol{c})$ measured at various temperatures are shown in Figs. 1 (a) and (b), respectively. The absorption tail for each polarization shifts to low-energy side with increasing temperature. At each temperature, the tail for $\boldsymbol{E} / / \boldsymbol{a}$ extends to lower energy than that for $\boldsymbol{E} / / \boldsymbol{c}$. The temperature coefficient of the energy shift is about $-1.0 \mathrm{meV} / \mathrm{K}$ for $\boldsymbol{E} / / \boldsymbol{a}$ and $-0.7 \mathrm{meV} / \mathrm{K}$ for $\boldsymbol{E} / / \boldsymbol{C}$ at the absorption coefficient $\alpha=10 \mathrm{~cm}^{-1}$.

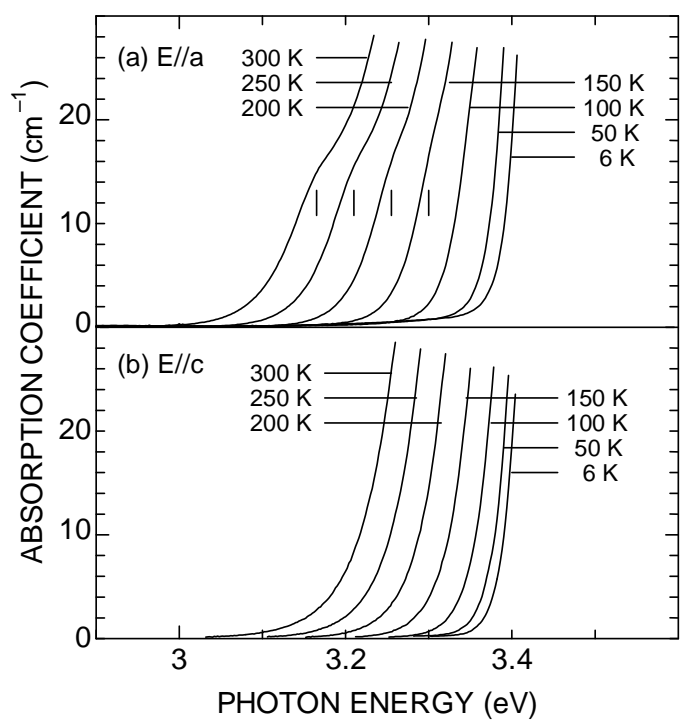

Figure 1 Absorption-edge spectra of $\mathrm{PbMoO}_{4}$ at various temperatures for (a) $\boldsymbol{E} / / \boldsymbol{a}$ and (b) $\boldsymbol{E} / / \boldsymbol{c}$.
For the polarization $\boldsymbol{E} / / \boldsymbol{a}$ in Fig. 1(a), no appreciable structure is observed in the absorption tail at $6 \mathrm{~K}$. When the temperature is raised to $150 \mathrm{~K}$, a weak absorption band appears as a shoulder at $3.30 \mathrm{eV}$ superimposed on the tail. The shoulder structure shows red shift and becomes remarkable with increasing temperature. At $300 \mathrm{~K}$, the peak position of the shoulder structure is located at around $3.16 \mathrm{eV}$.

The absorption tail for $\boldsymbol{E} / / \boldsymbol{c}$ in Fig. 1(b) also shows red shift as the temperature is increased. In contrast to the case of $\boldsymbol{E} / / \boldsymbol{a}$, no additional structure appears in the tail region at temperatures between 6 and $300 \mathrm{~K}$.

In Fig. 2 are plotted the absorption coefficients for $\boldsymbol{E} / / \boldsymbol{c}$ in a logarithmic scale vs. photon energy. The absorption coefficient at each temperature increases exponentially over about two orders of magnitude with increasing photon energy. The straight lines in Fig. 2 are fitted to the linear portions of the experimental curves. The solid lines for the temperatures at $100 \mathrm{~K}$ and above converge to a point. This fact indicates that the Urbach formula [16],

$$
\alpha=\alpha_{0} \exp \left(-\sigma \frac{E_{0}-E}{k_{\mathrm{B}} T}\right)
$$

holds for $\mathrm{PbMoO}_{4}$ crystals for $\boldsymbol{E} / / \boldsymbol{c}$, where $\alpha_{0}$ and $E_{0}$ are the parameters of the converging point, $\sigma$ the steepness parameter, $E$ the photon energy, $T$ the absolute temperature, and $k_{\mathrm{B}}$ the Boltzmann constant. The parameters of the converging point are obtained as $\alpha_{0}=1.5 \times 10^{4} \mathrm{~cm}^{-1}$ and $E_{0}=$ $3.52 \mathrm{eV}$.

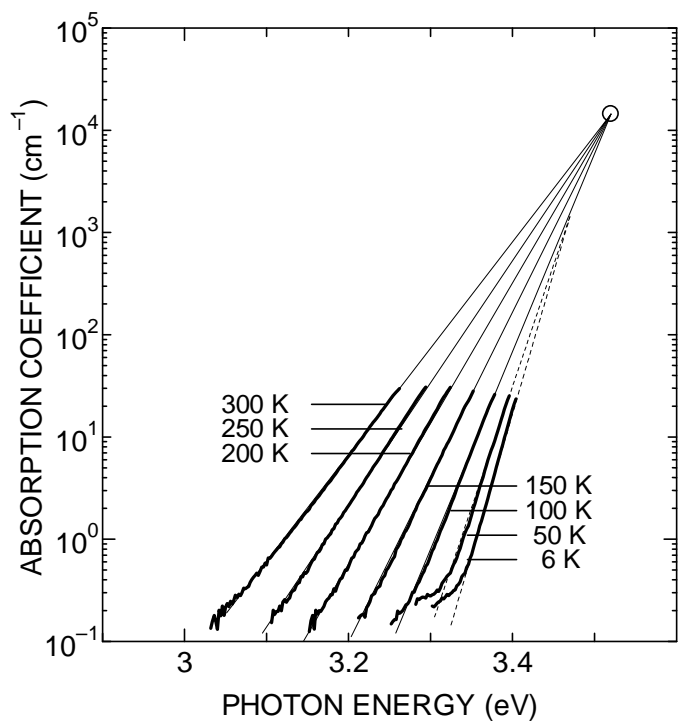

Figure 2 Logarithmic plot of the absorption spectra for $\boldsymbol{E} / / \boldsymbol{c}$ in Fig. 1(b) versus photon energy. The straight lines are the best fits of Eq. (1) to the thick curves of the experimental data.

The steepness parameters $\sigma$ at $T \geq 100 \mathrm{~K}$ are plotted by open circles in Fig. 3. The value of $\sigma$ is found to be temperature-dependent. This dependence is approximated by 


$$
\sigma=\sigma_{0} \frac{2 k_{\mathrm{B}} T}{\hbar \omega} \tanh \left(\frac{\hbar \omega}{2 k_{\mathrm{B}} T}\right)
$$

where $\sigma_{0}$ and $\hbar \omega$ stand for the high-temperature steepness parameter and the average energy of effective phonons interacting with excitons, respectively. The solid curve in Fig. 3 represents the best fit of Eq. (2) to the data points. From the fit, the parameters are estimated to be $\sigma_{0}=0.67$ and $\hbar \omega$ $=26 \mathrm{meV}$.

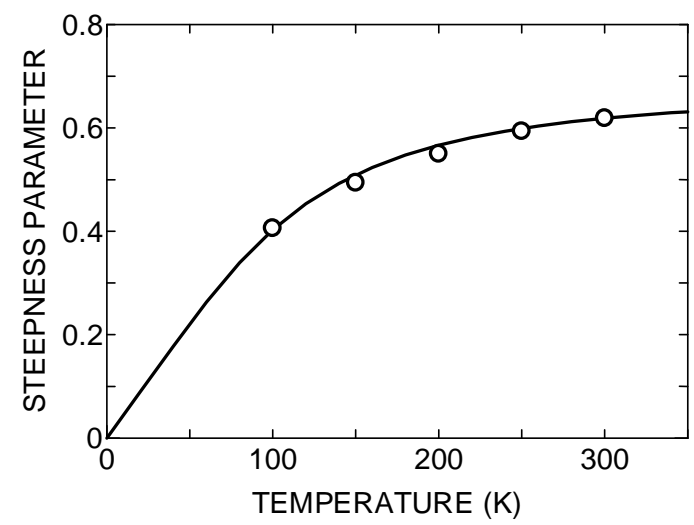

Figure 3 Temperature dependence of the steepness parameter $\sigma$ taken from the analysis of Fig. 2. The solid curve is the best fit according to Eq. (2).

4 Discussion Figure 1 shows that the intensity of the absorption tail for $\boldsymbol{E} / / \boldsymbol{a}$ is stronger than that for $\boldsymbol{E} / / \boldsymbol{c}$ at each temperature. The dichroism at the fundamental absorption edge of $\mathrm{PbMoO}_{4}$ has been observed at room temperature and $77 \mathrm{~K}$ by Bernhardt [5] and Groenink and Blasse [6], respectively, although detailed descriptions are not given in their papers. In the former, the absorption tail for $\boldsymbol{E} \perp \boldsymbol{c}$, which is equivalent to $\boldsymbol{E} / / \boldsymbol{a}$, extends to lower energy than that for $\boldsymbol{E} / / \boldsymbol{c}$. In the latter, the absorption intensity for $\boldsymbol{E} \perp \boldsymbol{c}$ is slightly stronger than that for $\boldsymbol{E} / / \boldsymbol{c}$ in the region above $3.3 \mathrm{eV}$. These results are in agreement with the present dichroism in Fig. 1. van Loo [4] has measured the temperature dependence of the absorption tail of $\mathrm{PbMoO}_{4}$. The polarization of light with respect to the crystal orientation is not described. Although the absorption spectra in ref. [4] look like our result for $\boldsymbol{E} / / \boldsymbol{c}$ rather than $\boldsymbol{E} / / \boldsymbol{a}$, it is supposed that the absorption for $\boldsymbol{E} / / \boldsymbol{a}$ is somewhat mixed with that for $\boldsymbol{E} / / \boldsymbol{c}$ in his spectra, as discussed later. The present result of the polarization dependence at the fundamental absorption edge is consistent with the result of our recent study, in which the intensity of the exciton transition for $\boldsymbol{E} / / \boldsymbol{a}$ is clarified to be much stronger than that for $\boldsymbol{E} / / \boldsymbol{c}$ [15].

It should be noted that the shoulder structure on the absorption tail for $\boldsymbol{E} / / \boldsymbol{a}$ in Fig. 1(a) shows significant red-shift and becomes remarkable with increasing temperature. In usual, an absorption band due to lattice defects hardly changes its peak position against temperature change. Such an absorption band located on the low-energy side of the intrinsic absorption tail becomes observable at low temperatures; in other words, it is buried in the tail part which expands to the low-energy side as the temperature is increased. The temperature dependence of the shoulder structure in Fig. 1(a), therefore, may suggest that this structure cannot be ascribed to such a usual defect band. It is worth noting that the exciton absorption band [15] and the shoulder structure in Fig. 1(a) are both observed distinctly for the polarization $\boldsymbol{E} / / \boldsymbol{a}$, while they are extremely weak or not discernible for $\boldsymbol{E} / / \boldsymbol{c}$. This correspondence suggests that the shoulder structure is attributed to the exciton absorption perturbed by some lattice defects, the transition probability of which increases with increasing temperature. Further investigation is necessary to clarify the origin of this structure. In ref. [5], a broad absorption band with a peak at around $3.2 \mathrm{eV}$ has been observed as a shoulder on the absorption tail. The intensity is stronger for $\boldsymbol{E} \perp \boldsymbol{c}$ than $\boldsymbol{E} / / \boldsymbol{c}$. It is uncertain whether this band is the same as the shoulder structure discussed above.

Fortunately, the absorption tail for $\boldsymbol{E} / / \boldsymbol{c}$ is free from the defect-related band. The result in Fig. 2 clearly indicates that the Urbach rule of Eq. (1) holds for the tail for $\boldsymbol{E} / / \mathbf{c}$ in the temperature range $T \geq 100 \mathrm{~K}$. In general, the converging point of the straight lines fitted to the logarithmic plot of the Urbach tails is located around the peak position of the lowest exciton band $[16,18]$. In the $\mathrm{PbMoO}_{4}$ crystals, the intensity of the exciton transition for $\boldsymbol{E} / / \boldsymbol{c}$ is very weak and the clear exciton peak is hardly observable at around the converging point $E_{0}=3.52 \mathrm{eV}$ [15]. This is consistent with the present result that the absorption coefficient at the converging point $\left(\alpha_{0}=1.5 \times 10^{4} \mathrm{~cm}^{-1}\right)$ is significantly small compared to the values in alkali halides, for instance, $\alpha_{0}=6 \times 10^{9} \mathrm{~cm}^{-1}$ in $\mathrm{KBr}$ [19]. van Loo has obtained the values of the parameters of the converging point as $\alpha_{0}=$ $1.0 \times 10^{5} \mathrm{~cm}^{-1}$ and $E_{0}=3.57 \mathrm{eV}$ [4], although the convergence of the lines fitted to the logarithmic plot of the absorption coefficients seems not so good as the present result in Fig. 2. The value of $\alpha_{0}$ in his result is about one order of magnitude larger than our value for $\boldsymbol{E} / / \boldsymbol{c}$. This discrepancy is probably ascribed to the mixing of the absorption spectra of $\boldsymbol{E} / / \boldsymbol{a}$ and $\boldsymbol{E} / / \boldsymbol{c}$ in ref. [4], since the absorption coefficient for $\boldsymbol{E} / / \boldsymbol{a}$ is much greater than that for $\boldsymbol{E} / / \boldsymbol{c}$. In the analysis of the Urbach tail in $\mathrm{PbWO}_{4}$ for the polarization $\boldsymbol{E} / / \boldsymbol{a}$ [17], we obtained the absorption coefficient $\alpha_{0}$ $=4.2 \times 10^{7} \mathrm{~cm}^{-1}$ at the converging point. This value is fairly larger than that in $\mathrm{PbMoO}_{4}$, reflecting the strong exciton transition in $\mathrm{PbWO}_{4}$ [20].

The temperature dependence of the steepness parameter $\sigma$ is described by Eq. (2), as shown in Fig. 3. The optical phonon energies in $\mathrm{PbMoO}_{4}$ are found in a wide range between 7 and $104 \mathrm{meV}[21,22]$. The value of $\hbar \omega=26 \mathrm{meV}$ estimated from the fitting in Fig. 3 could be regarded as an average of these phonon energies.

According to ref. [18], the value of the hightemperature steepness parameter $\sigma_{0}$ is inversely proportional to the strength of the exciton-phonon coupling. In 
three-dimensional lattices, an exciton becomes self-trapped and gives rise to a luminescence band with large Stokes shift, when the value of $\sigma_{0}$ is smaller than the critical value $\sigma_{\mathrm{c}}=1.50$ [18]. The present value of $\sigma_{0}=0.67$ is considerably smaller than this critical value, indicating that the exciton-phonon coupling is strong in $\mathrm{PbMoO}_{4}$. It is well known $[4,6]$ that the intrinsic luminescence due to self-trapped excitons in $\mathrm{PbMoO}_{4}$ is observed with a peak at around 2.3 $\mathrm{eV}$ when excited with light of the photon energies above $3.3 \mathrm{eV}$. The large Stokes shift $(\sim 1.0 \mathrm{eV})$ of the intrinsic luminescence band is in satisfactorily agreement with the above conclusion on the strong exciton-phonon coupling in $\mathrm{PbMoO}_{4}$.

5 Conclusion In the present study it was shown that the absorption tail of $\mathrm{PbMoO}_{4}$ crystals is strongly dependent on the polarization of light. The presence of lattice defects which result in an anisotropic optical absorption band superimposed on the absorption tail was found for the polarization $\boldsymbol{E} / / \boldsymbol{a}$. It was confirmed that the Urbach rule holds for $\boldsymbol{E} / / \boldsymbol{c}$. The obtained values of the Urbach parameters were fairly consistent with other experimental results, i.e., exciton transition and luminescence properties. We believe that the present result on the Urbach rule is more reliable than the earlier result in ref. [4].

Acknowledgements The authors are indebted to Mr. H. Mitani and Mr. T. Aoki for their assistance in the experiments. This work was supported by the Joint Studies Program of the Institute for Molecular Science.

\section{References}

[1] D. A. Pinnow, L. G. Van Uitert, A. W. Warner, and W. A. Bonner, Appl. Phys. Lett. 15, 83 (1969).

[2] S. Takano, S. Esashi, K. Mori, and T. Namikata, J. Crystal Growth 24/25, 437 (1974).

[3] M. Minowa, K. Itakura, S. Moriyama, and W. Ootani, Nucl. Insturum. Methods Phys. Res. A 320, 500 (1992).

[4] W. van Loo, Phys. Status Solidi A, 27, 565 (1975).

[5] H. J. Bernhardt, Phys. Status Solidi A 45, 353 (1978).

[6] J. A. Groenink and G. Blasse, J. Solid State Chem., 32, 9 (1980).

[7] H. C. Zeng, J. Crystal Growth 171, 136 (1997).

[8] T. M. Bochkova, M. D. Volnyanskii, D. M. Volnyanskii, and V. S. Shchetinkin, Phys. Solid State, 45, 244 (2003).

[9] Sangeeta, D. G. Desai, A. K. Singh, M. Tyagi, and S. C. Sabharwal, J. Crystal Growth 296, 81 (2006).

[10] J. Chen, T. Liu, D. Cao, and G. Zhao, Phys. Status Solidi B 245, 1152 (2008).

[11] V. Babin, P. Bohacek, E. Bender, A. Krasnikov, E. Mihokova, M. Nikl, N. Senguttuvan, A. Stolovits, Y. Usuki, and S. Zazubovich, Radiat. Meas. 38, 533 (2004).

[12] D. A. Spassky, S. N. Ivanov, V. N. Kolobanov, V. V. Mikhailin, V. N. Zemskov, B. I. Zadneprovski, and L. I. Potkin, Radiat. Meas. 38, 607 (2004).

[13] J. Leciejewicz, Z. Kristallogr. 121, 158 (1965).
[14] R. M. Hazen, L. W. Finger, and J. W. E. Mariathasan, J. Phys. Chem. Solids, 46, 253 (1985).

[15] M. Fujita, M. Itoh, H. Mitani, Sangeeta, and M. Tyagi, Phys. Status Solidi B (DOI: 10.1002/pssb.200945447).

[16] M. V. Kurik, Phys. Status Solidi A 8, 9 (1971).

[17] M. Itoh, H. Yokota, M. Horimoto, M. Fujita, and Y. Usuki, Phys. Status Solidi B 231, 595 (2002).

[18] M. Schreiber and Y. Toyozawa, J. Phys. Soc. Jpn. 51, 1544 (1982).

[19] T. Tomiki, T. Miyata, and H. Tsukamoto, Z. Naturforsch. A 29, 145 (1974).

[20] M. Fujita, M. Itoh, M. Horimoto, and H. Yokota, Phys. Rev. B 65, 195105 (2002).

[21] K. Kesavasamy and N. Krishnamurthy, Can. J. Phys. 60, 1447 (1982).

[22] T. Sinagawa, J. Suda, T. Sato, and H. Saito, J. Phys. Soc. Jpn. 69, 464 (2000). 